\title{
Noise parametric identification and whitening for LIGO 40-meter interferometer data
}

\author{
Elena Cuoco, Giovanni Losurdo \\ INFN Firenze Section \\ Giovanni Calamai \\ Osservatorio Astrofisico di Arcetri and INFN Firenze Section \\ Leonardo Fabbroni, Massimo Mazzoni, Ruggero Stanga \\ Dipartimento di Astronomia e Scienze dello Spazio, Universitá di Firenze and INFN Firenze section \\ Gianluca Guidi, Flavio Vetrano \\ Universita' di Urbino and INFN Firenze Section
}

(September 22, 2018)

\begin{abstract}
One of the goal of the gravitational data wave analysts is the knowledge and the accurate estimation of the noise power spectral density of the data taken by the detector, being it necessary in the detection algorithms. In this paper we showed how it is possible estimate the noise power spectral density of gravitational waves detectors using modern parametric techniques and how it is possible whiten the noise data before pass it to the algorithms for the gravitational waves detection. We report the analysis we made on data taken by Caltech 40-meter prototype interferometer to identify its noise power spectral density and to whiten the sequence of noise. We concentrate our study on data taken in November 1994, in particular we analyzed two frames of data: the 18nov94.2.frame and the 19nov94.2.frame. We show that it is possible to whiten these data, to a good degree of whiteness, using a high order whitening filter. Moreover we can choose to whiten only restricted band of frequencies around the region we are interested in, obtaining a higher level of whiteness.
\end{abstract}

04.80.Nn, 07.05Kf, 07.60Ly, 05.40Ca, 05.40C

\section{INTRODUCTION}

The building of large interferometers is going to reach the phase of data taking: TAMA (Japanese) [1] is already working; GEO (British/German) 2 will begin to take data next year; LIGO (U.S.A.) [3] 近 in 2002; VIRGO (French/Italian) [5] in 2003 (see e.g. [6] [7] [8] for general and exhaustive description of interferometric Gravitational Waves detectors).

The large amount of data produced by gravitational wave detectors will be essentially noise and, hopefully, buried in noise there will be the signal we are looking for. Ground-based interferometric detectors are sensible to a broad band frequencies $(2-3 \mathrm{~Hz}$ to more than $1 \mathrm{kHz})$ in revealing relative displacement of test masses at the near and far extremities of interferometer arms due to GW signal, but unfortunately a lot of other factors can cause a similar displacement. The test masses are suspended to pendular structures in order to isolate them from seismic noise [9], but thermal noise of the suspension chain will cause a displacement of the mass [10]. Also shot noise and radiation pressure of the laser will move the mirrors [6] [11. The physicists are working in modeling all possible causes of noise in the interferometer giving out a sensitivity curve of the apparatus [12] 13] 14] 15] 16] 18].

This curve is limited at low frequencies by seismic noise; in the middle band by thermal noise and at high frequencies (higher than $0.7-1 \mathrm{kHz}$ ) by shot-noise. The sensitivity curve of these detector is a broad-band noise plus several very narrow peaks due to the violin modes of the suspensions wires, that will make the detection of a gravitational signal in this frequency band very difficult. For this reason efforts have been made in the preparation of the analysis of data for cutting [19] [20] [21] out these resonances.

The analysis of data to detect the gravitational signal requires an accurate knowledge of the noise, which means a statistical characterization of the stochastic process, and in the case of local stationarity and Gaussian nature an accurate estimation of the Power Spectral Density (PSD).

Moreover if the output of the interferometer will be non stationary over a long period of time, we must be able in following the changes in the PSD. A way to achieve this is to estimate the PSD on a chunk of data at different interval of time, using classical techniques [24] [25] [26].

We proposed the use of adaptive methods to follow on line the change in the feature of the spectrum in such a way to have at any desired instant the correct curve for the PSD [27].

If we are able in identifying the noise of our detector we can also apply the whitening procedure of the data.

The goal of a whitening procedure is to make the sequence of data delta-correlated, removing all the correlation of the noise. Most of the theory of detection is in the frame of a wide-sense stationary Gaussian white noise, but in our problem the noise is surely a colored one and, in principle, there could be present non stationary and non Gaussian features. If we whiten the data, supposing 
hence to be in the frame of a stationary and Gaussian noise, we can apply the optimal algorithm detection 28.

Moreover when we are searching a transient signal of unknown form it is very important to have a whitened noise [29]. The importance of whitening data is also linked to the possibility of reducing their dynamical range [31] [30].

In this paper we show how to identify and how to whiten the noise data produced by LIGO-40 meter interferometric detector before applying any algorithm detection.

The data taken from Caltech 40-meter prototype interferometer in November 1994 [22] [23] have been given from the LIGO collaboration at disposal of the data analysis groups of other experiments to perform some test of data analysis algorithms. A typical PSD of the output of LIGO-40 meter data is plotted in figure 1. It is characterized by a huge number of spectral lines. Part of them are due to the violin modes, part to the laser noise and mostly of them to the $60 \mathrm{~Hz}$ and its harmonics power supply.

We use these data to check the algorithms of parametric noise identification and the algorithms which perform the whitening on realistic data taken by an interferometric gravitational waves detector, having already analyzed and tuned these techniques on Virgo-like simulated data (see reference [27]).

The concepts and the notation in this paper are described in detail in our earlier work [27]. Sections II and III give a brief summary of the methods, but the reader is directed to Ref. 27] for details.

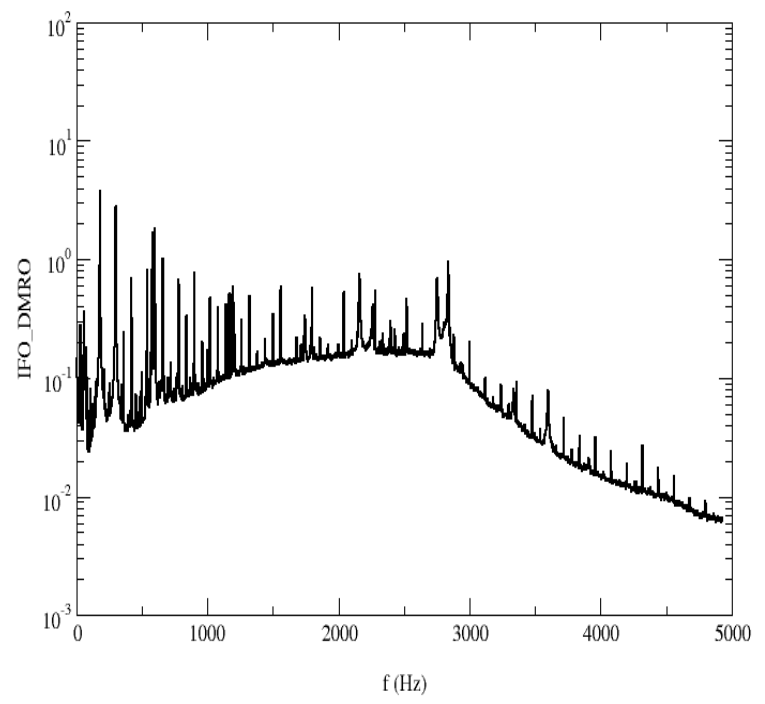

FIG. 1. Example of PSD of LIGO 40-meter data

\section{PARAMETRIC MODELING AND WHITENING}

The advantages of power spectral parametric estimation with respect to the classical spectral methods are described in an exhaustive way in reference 24. We want to underline that one of its advantage in linked to the better spectral resolution we obtain if we suppose that the process we are analyzing is governed by a dynamical law, because we can use the estimation of autocorrelation function until a certain lag and then extrapolate its value at successive lags, under the dynamical hypothesis we made.

An other advantage is that we may compress the information of the PSD in a restricted number of parameters (the parameters of our model) and not in the full autocorrelation function. This can help us, for example, if we want to create a data base of noise sources.

In this paper we analyze only the Auto-Regressive (AR) parametric modeling because, as a byproduct, it offers the possibility to write down a linear whitening filter [27].

\section{A. AR models}

An Auto-Regressive process $x[n]$ of order $P(A R(P))$ is governed by the relation

$$
x[n]=-\sum_{k=1}^{p} a[k] x[n-k]+w[n],
$$

which links the value of the data $x[n]$ to the previous $P$ ones by the AR parameters $a[k]$, being $w[n]$ a Gaussian white noise process. The theoretical form of the PSD for such a process is given by

$$
P_{A R}(f)=\frac{\sigma^{2}}{\left|1+\sum_{k=1}^{P} a_{k} \exp (-i 2 \pi k f)\right|^{2}},
$$

being $\sigma$ one of the parameters to be estimated linked to the RMS of the process.

The relationship between the parameters $a[k]$ of the AR model and the autocorrelation function $r_{x x}(n)$ of the process $x[n]$ is given by the Yule-Walker equations

$$
r_{x x}[k]= \begin{cases}-\sum_{l=1}^{p} a_{l} r_{x x}[k-l] & \text { for } k \geq 1 \\ -\sum_{l=1}^{p} a_{l} r_{x x}[-l]+\sigma^{2} & \text { for } k=0 .\end{cases}
$$

The problem of determining the AR parameters is the same of that of finding the optimal "weights vector" $\mathbf{w}=w_{k}$, for $k=1, \ldots P$ for the problem of linear prediction 24]. In the linear prediction we would predict the sample $x[n]$ using the $P$ previous observed data $\mathbf{x}[n]=\{x[n-1], x[n-2] \ldots x[n-P]\}$ building the estimate as a transversal filter: 


$$
\hat{x}[n]=\sum_{k=1}^{P} w_{k} x[n-k] .
$$

We choose the coefficients of the linear predictor by minimizing a cost function that is the mean squares error $\epsilon=\mathcal{E}\left[e[n]^{2}\right]$, being

$$
e[n]=x[n]-\hat{x}[n]
$$

the error we make in this prediction, obtaining the so called Normal or Wiener-Hopf equations

$$
\epsilon_{\min }=r_{x x}[0]-\sum_{k=1}^{P} w_{k} r_{x x}[-k]
$$

which are identical to the Yule-Walker ones with

$$
\begin{aligned}
w_{k} & =-a_{k} \\
\epsilon_{\text {min }} & =\sigma^{2}
\end{aligned}
$$

This relationship between AR model and linear prediction assures us to obtain a filter which is stable and causal 24]: this is the fundamental relation in building our whitening filter.

A method of solving the Yule-Walker equation is the Durbin algorithm 32].

The strategy of this method is the knowledge of the optimal $(P-1)$ th order filter to calculate from it the optimal $P$ th order.

\section{B. Link between AR model and whitening filter}

When we find the AR $P$ parameters that fit a PSD of a noise process, what we are doing is to find the optimal vector of weights that let us reproduce the process at the time $n$ knowing the process at the $P$ previous time. All the methods that involves this estimation try to make the error signal (see equation (5) ) a white process in such a way to throw out all the correlation between the data (which we use for the estimation of the parameters). So one of the output of the filter which identifies the noise will be the whitened part of the data.

The Durbin algorithm, used to estimated the parameters $a[k]$, introduces in a natural way the Lattice structure for the whitening filter 32]. The equations which represent our lattice filter in the time domain could be written

$$
\begin{aligned}
& e_{p}^{f}[n]=e_{p-1}^{f}[n]+k_{p} e_{p-1}^{b}[n-1] \\
& e_{p}^{b}[n]=e_{p-1}^{b}[n-1]+k_{p} e_{p-1}^{f}[n]
\end{aligned}
$$

being $e_{p}^{b}$ the backward error, that is the error we make, in a backward way, in the prediction of the data $x[n-p+1]$ using $p-1$ successive data, and $e_{p}^{f}$ the forward error, that is the error we made in estimating the data $x[n]$ using the $P$ previous data. The coefficients $k_{p}$ are the so-called reflection or PARCOR coefficients, being linked to the correlation that the data $x[n]$ has with the $x[n-p]$, neglecting the $p-1$ values of $x$ in between. In figure 2 is showed how the lattice structure is used to estimate the forward and backward errors (see reference 27]).
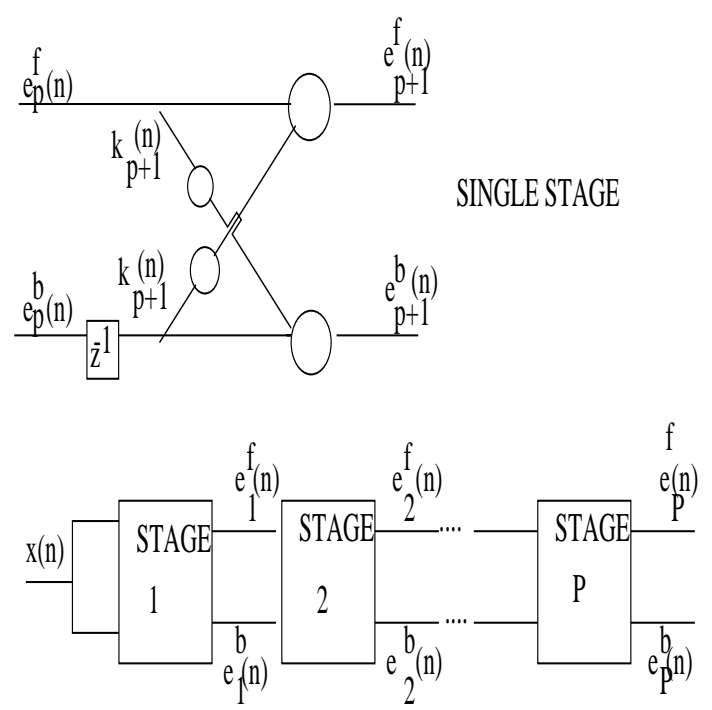

FIG. 2. Lattice structure for Durbin filter.

Using a lattice structure we can implement the whitening filter following these steps:

- estimate the values of the autocorrelation function $\hat{r}_{x x}[k], 0 \leq k \leq P$ of our process $x[n]$

- use the Durbin algorithm to find the reflection coefficients $k_{p}, 1 \leq p \leq P$;

- implementation of the lattice filter with these coefficients $k_{p}$ initiating the filter $e_{0}^{f}[n]=e_{0}^{b}[n]=x[n]$.

In this way the forward error at the stage $P$-th is equivalent to the forward error of a transversal filter and represents the output of the whitening filter.

The procedure of whitening will be accomplished before applying the algorithms for the detection of gravitational signal of different wave forms. The level of whiteness of the data needed for the various algorithms could be different. We want now to introduce a parameter that let us quantify the level of whiteness of data at the output of whitening filter.

\section{The "whiteness" of data: measure of flatness of PSD} 24

The spectral flatness measure for a PSD is defined as

$$
\xi=\frac{\exp \left(\frac{1}{N_{s}} \int_{-N_{s} / 2}^{N_{s} / 2} \ln (P(f)) d f\right)}{\frac{1}{N_{s}} \int_{-N_{s} / 2}^{N_{s} / 2} P(f) d f}
$$


where the integral is extended in the bandwidth of Nyquist frequency; this parameter satisfies

$$
0 \leq \xi \leq 1
$$

If $P(f)$ is very peaky, then $\xi \simeq 0$, if $P(f)$ is flat than $\xi=1$.

Using the definition (11) the flatness for a process at the output of a whitening filter built with a minimum phase filter (as the AR filter is) is

$$
\xi_{e}=\xi \frac{r_{x x}[0]}{r_{e e}[0]}
$$

where $r_{x x}[0]$ and $r_{e e}[0]$ are the values of the autocorrelation function of the process before and after the whitening procedure, and $\xi$ is the value of flatness for the initial sequence [24].

\section{Order Selection}

The idea of the whitening filter is that the process we analyze is an autoregressive one and that once we have the AR parameters we can use them in the whitening filter.

In general we don't know the order of our process, even if we suppose that it is an AR one. If it is an AR of order $\mathrm{P}$, and we use an order $p<P$, the fitted spectrum will be smoother than the original one; if we choose an order $p>P$, there may be spurious peaks in the spectrum. In both cases the whitening will be not good.

If our process is not $\mathrm{AR}$, the number of parameters could be in principle infinite. We must then fix a criterion that let us select the right order of the process, or at least the best one.

We used the classical order selection criteria 24] 25] [33], that is the Akaike information criterion (AIC), the forward prediction error (FPE) the Parzen's criterion (CAT) and the minimum description length (MDL) one

$$
\begin{aligned}
A I C(P) & =N \log \epsilon(P)+2 P, \\
F P E(P) & =\epsilon(P) \frac{N+P+1}{N-P-1}, \\
C A T(P) & =\left(\frac{1}{N} \sum_{j=1}^{P} \frac{N-j}{N \epsilon_{j}}\right)-\frac{N-P}{N \epsilon_{P}}, \\
M D L(P) & =N \log \epsilon(P)+P \log N,
\end{aligned}
$$

where $\epsilon(P)$ is the mean square error at the order $P$ and $N$ is the length of data. In literature the MDL criterion is considered the best among them, because it is robust with respect to the length of the sequence, while the others depend a lot on $\mathrm{N} 25$.

\section{ADAPTIVE METHODS:LEAST SQUARES LATTICE}

The implementation of an adaptive filter follows two steps: the filtering of the input data and the adjustment of the filter parameters with which we process the data to the next iteration. The filters parameters are updated by minimizing a cost function. The way in which we build this cost function distinguishes the adaptive methods [32] 34. The Least Squares based methods build their cost function using all the information contained in the error function at each step, writing it as the sum of the error at each step up to the iteration $n$ :

$$
\epsilon[n]=\sum_{i=1}^{n} \lambda^{n-i} e^{2}(i \mid n),
$$

being

$$
e(i \mid n)=d[i]-\sum_{k=1}^{N} x_{i-k} w_{k}[n],
$$

where $d$ is the signal to be estimated, $x$ are the data of the process and $w$ the weights of the filter. We introduced the forgetting factor $\lambda$ that let us tune the learning rate of the algorithm. This coefficient can help when there are non stationary data in the sequence and we want that the algorithm have a short memory. If we have stationary data we fix $\lambda=1$.

There are two ways to implement the Least Squares methods for the spectral estimation: in a recursive way (Recursive Least Squares or Kalman Filters) or in a Lattice Filters using fast techniques [27] [32]. The first kind of algorithm, examined in [35], has a computational cost proportional to the square of the order of filter, while the cost of the second one is linear in the order $P$. We will report only the application of Least Squares Lattice (LSL) algorithm on LIGO 40-meters data, because in our previous work 27 the authors showed that this is the best choice among the adaptive algorithms the authors analyzed for the problem we have to face.

\section{RESULTS ON 40-METER LIGO DATA}

We use two frames of the LIGO 40-meter interferometer data: the 18nov.2.frame and the 19nov.2.frame. We use the GRASP interface to read the data using only the frames taken with the interferometer locked [30] 36]. We are not interested to the absolute sensitivity of such interferometer, but only in the features of the Power Spectral Density, so we don't use calibrated data, and we analyzed the IFO output of the data in ADC counts. This is a fast channel which was sampled at about $10 \mathrm{kHz}$.

In figure 1 a typical power spectral density of these data is displayed: it is characterized by a high number of 
lines, which could be mainly harmonics of the electromagnetic interference at $60 \mathrm{~Hz}$, by two broad peaks around $180 \mathrm{~Hz}$ and $300 \mathrm{~Hz}$ which could be due to the laser power supply, by the violin resonances around $500-600 \mathrm{~Hz}$, and a continuous background, due to electronic noise, shot noise, seismic noise etc.

It is evident that we would need a high number of parameter to fit the spectrum and to build the respective whitening filter.

\section{A. 18nov94.2.frame}

We try to obtain an estimate of the order we need for whitening the noise PSD of this frame. The MDL criterion gives an order of 515. As first test we use this value to whiten the 18nov94.2.frame data.

In figure 3 we plotted the PSD of 18nov94.2.frame averaged on 30 sequence of data of $\sim 6 \mathrm{~s}$ in input to the Durbin and LSL whitening filters. In the same figure we can see the AR(515) fit to the PSD and the PSD at the output of the whitening filters.

In the first figure it is evident that the filters succeed in whitening the overall behavior of the noise power spectrum, but not in eliminating all the spectral lines.

Besides the several harmonics spectral lines, the main problem in the whitening of these data is linked to the peaks in the range of frequencies from 0 to $1000 \mathrm{~Hz}$. These peaks are broad, and it is difficult to fit them with an allpoles models as it is evident in the zoom we made in this band of frequencies in figure 3 .
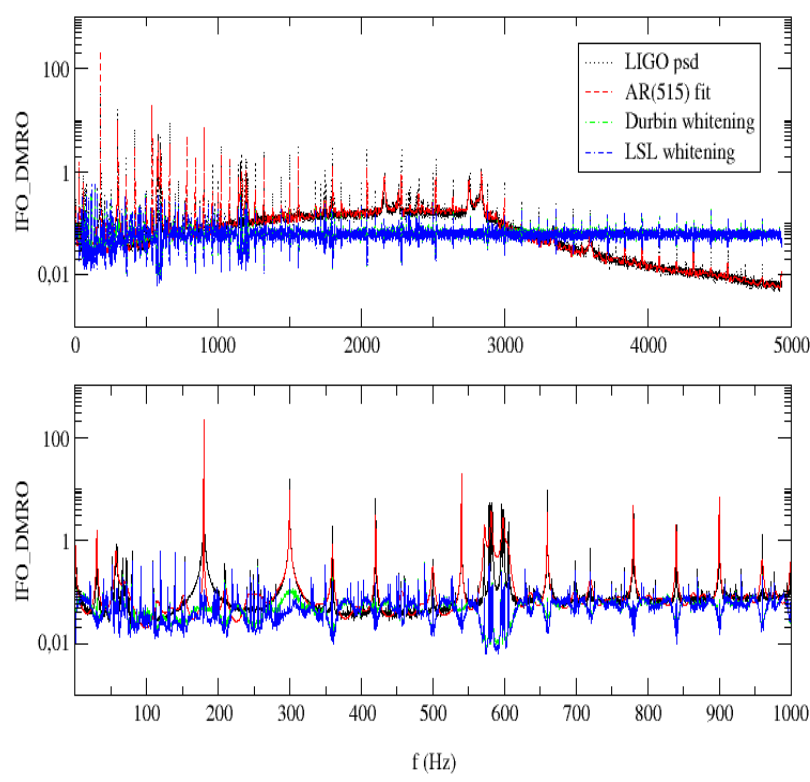

FIG. 3. Application of AR(515) whitening filter

Notwithstanding these problems, the values of flatness at the output of whitening filters is quite good as we see in figure 4 and in table

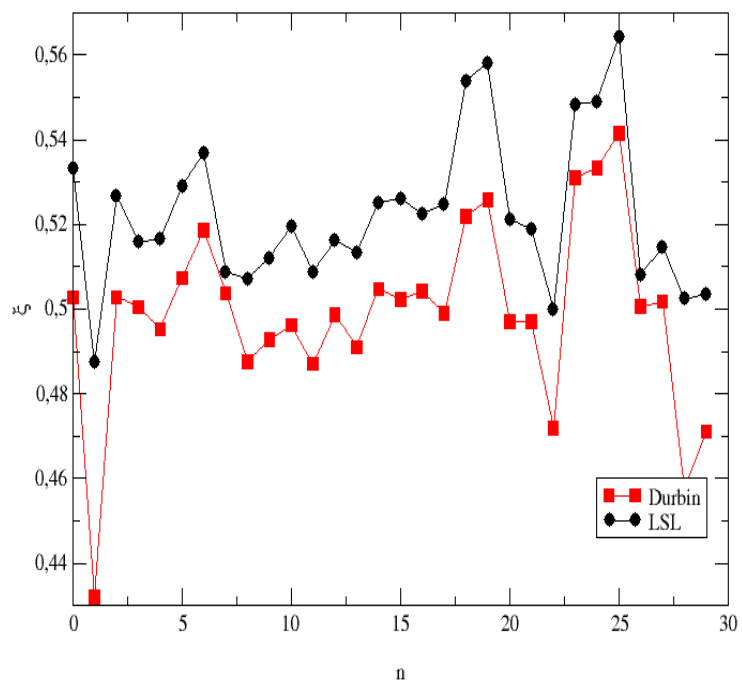

FIG. 4. Flatness at the output of Durbin and LSL whitening filter of order $\mathrm{P}=515$.

\begin{tabular}{|c|r|r|}
\hline \hline 18nov94.2 & Durbin & LSL \\
\hline \hline 0.0220 & 0.877 & 0.917 \\
\hline \hline
\end{tabular}

TABLE I. Values of flatness on averaged psd before and after the whitening filters. 
This is reflected in the time-domain output of the filter where it is evident that the rms of the data is remarkably reduced (see figure 5 ).

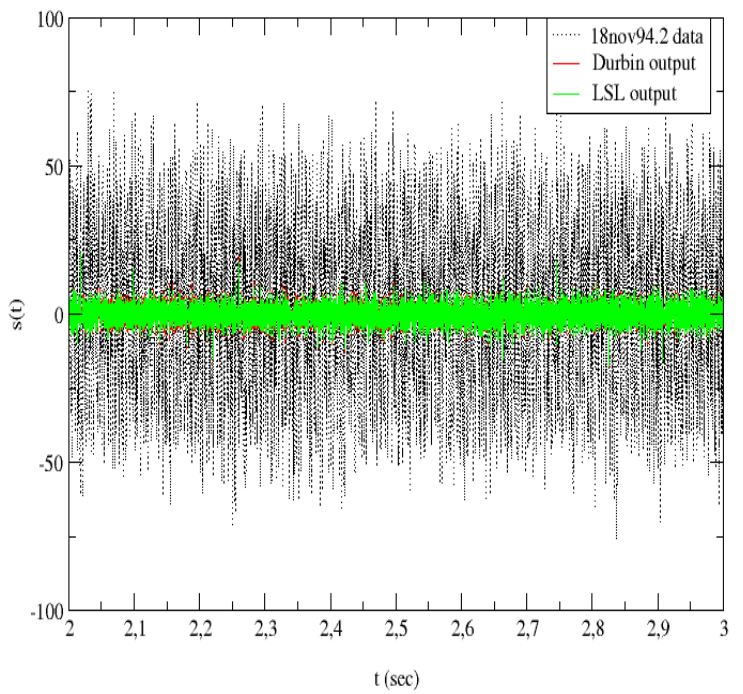

FIG. 5. Time-domain input and outputs of whitening filters.

It is to be noted that the results of the adaptive algorithm reported in figure 4 and in table 10 are a little better that those of the 'static' Durbin algorithm. These can be explained by the fact that the estimation of parameter of Durbin whitening needs a previous estimation of autocorrelation function, while the adaptive LSL filter estimate the parameters directly by the time data, avoiding the problem of errors propagation. Moreover in those runs the adaptive algorithm was tuned in such a way to have a long memory to let the filter continue to adjust its parameters with the input of new data.

\section{Whitening filter of higher order}

Until now we used the order of whitening filter as the one estimate on the averaged PSD or autocorrelation function. Now we run the order selection criteria on a single sequence of data long $\sim 6 \mathrm{~s}$. The results are reported in table II.

\begin{tabular}{|c|r|r|r|}
\hline \hline MDL & AIC & FPE & CAT \\
\hline \hline 662 & 2682 & 2682 & 2682 \\
\hline \hline
\end{tabular}

TABLE II. Minimum of order selection criteria on a single sequence of data for the 18nov94.2.frame.

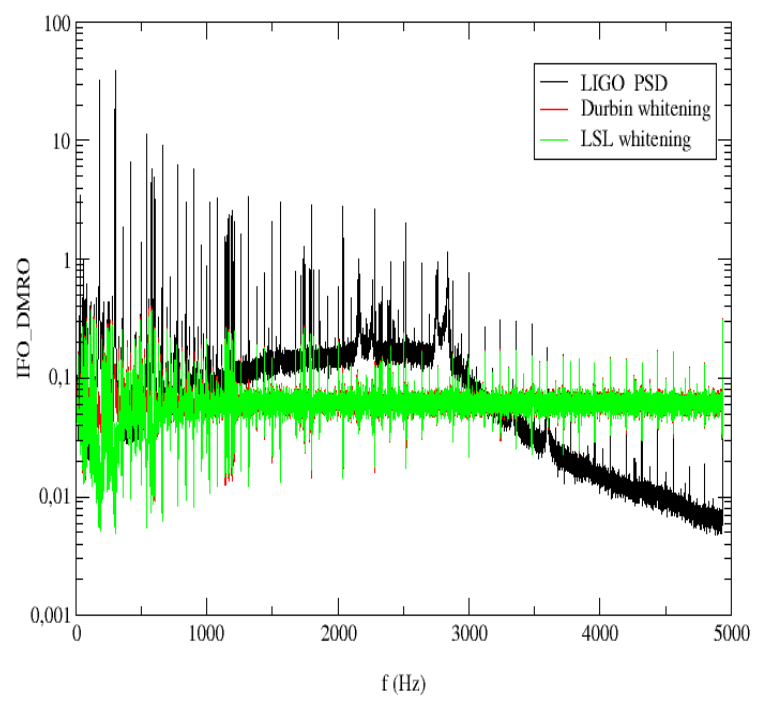

FIG. 6. Whitening of 18nov94.2.frame data with a filter of order $\mathrm{P}=662$. PSD averaged on 30 sample of $6 \mathrm{sec}$.

We try the whitening procedure using firstly a filter of $P=662$ coefficients and then a filter of $\mathrm{P}=2682$.

In figure 6 we report the PSD at the output of whitening filter of order $P=662$ as averaged on 30 samples of $6 \mathrm{~s}$ of data. As it is evident there exists always the problem in the low frequency part of the spectrum due to the two broad peaks. A typical value of the flatness on one sample of data is $\sim 0.5$ for the Durbin filter and $\sim 0.55$ for the LSL one.

In figure 7 we plotted the outputs of whitening filters with $P=2682$ as suggested from the AIC, FPE and CAT criteria. The higher order filters succeeded in eliminating the narrow spectral lines at higher frequencies, but the problems in the band under $1000 \mathrm{~Hz}$ still remain. The flatness of the output PSD is remarkably better in this case. A typical value for the Durbin whitening filter is $\xi \sim 0.6$, while for the LSL one is $\xi \sim 0.7$ 


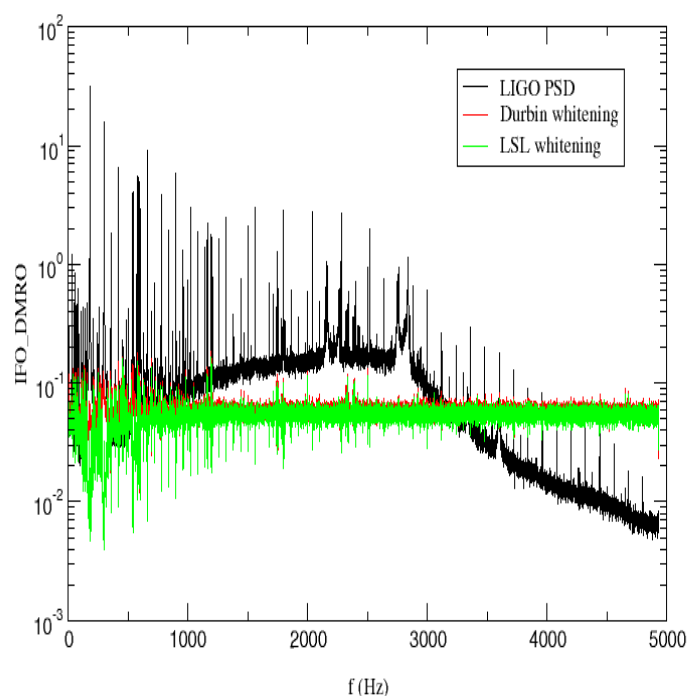

FIG. 7. Whitening of the 18nov94.2.frame with 2682 parameters.

\section{Whitening the data on a restricted band of frequencies.}

By considering the fact that the signal detection algorithm will act on restricted band of frequencies, where it is most probable to find a gravitational signals and that the whitening procedure will be limited to an interval of frequencies and not on all the Nyquist interval, we try to whiten the 18nov94.2.frame on the band of frequencies from 0 to $1234 \mathrm{~Hz}$, where the algorithms have met with major difficulties in whitening the noise spectrum. To decimate the set of data at our disposal we let the data pass through a Butterworth low pass filter of 4 order that cuts off all the frequencies over $1234 \mathrm{~Hz}$ and then we re-sample the data with a sampling factor 4 .

The output of this pre-filter procedure has been sent to the whitening filter. We estimate the best order for the whitening filter using a single realization of $6 \mathrm{~s}$ of data and applying to it the order selection criteria (see table III).

\begin{tabular}{|c|r|r|r|}
\hline \hline MDL & AIC & FPE & CAT \\
\hline \hline 329 & 1154 & 1154 & 1072 \\
\hline \hline
\end{tabular}

TABLE III. Order selection criteria for 18nov94.2.frame on the band $0-1233.5 \mathrm{~Hz}$.
We find again that the MDL gives the minimum value for $P$. We whiten the data both with the value found by the MDL criterium, that is $P=329$, and with the value $P=1154$ which is the value found by the AIC and FPE criteria.

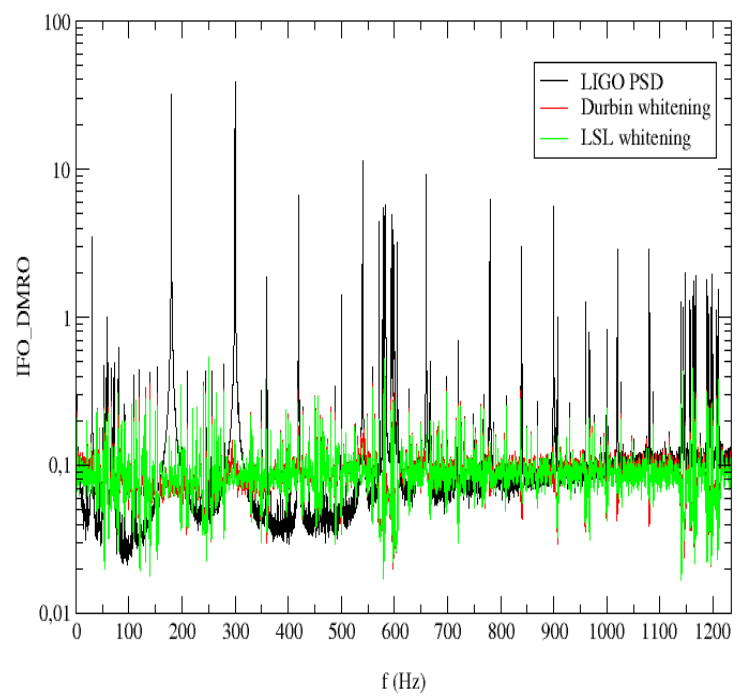

FIG. 8. PSD at the output of whitening filters with $P=329$ for 18 nov94.2. frame data in the band $0-1233.5 \mathrm{~Hz}$.

The PSD at the outputs of whitening filters with $P=$ 329 are plotted in figure 8 .

Even if we have restricted the band of interest the difficulties of whitening the broad peaks remain. Moreover the band of frequencies around $600 \mathrm{~Hz}$ is characterized by several spectral lines plus some broad peaks, due to violin resonances [19]. Most of the parameters of the whitening fit try to cancel out the broad peaks, so the whitening is worse also on narrow lines. Perhaps it could be useful separate the whitening in two steps, eliminate the narrow lines and whiten the broad peaks or augmenting the number of parameters. In fact if we use $P=1154$ the whitening we obtain in this band of frequencies is good as you can see in figure 9 . 


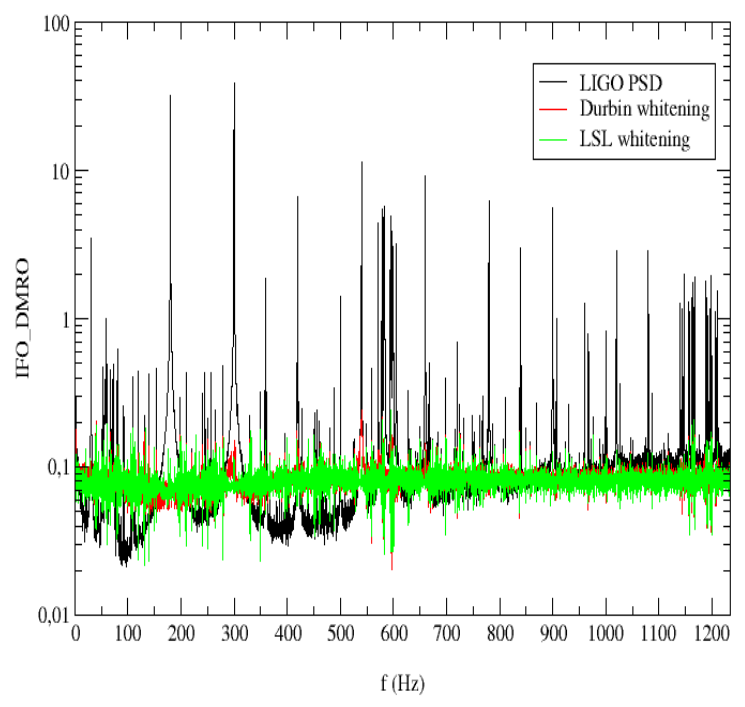

FIG. 9. PSD at the output of whitening filters with $P=1154$ for 18 nov94.2.frame data in the band $0-1233.5 \mathrm{~Hz}$.

The typical value of the flatness for the whitening with $P=1154$ is between 0.6 and 0.8 and the Durbin filter and LSL filter have similar performance.

Moreover the choice of order of the filter is not tied up by the order selection criteria.

As we have already outlined [27] this number is an indicative one; the selection criteria depend also on the length of sample to analyze. If we use for example frame of data long $26 \mathrm{~s}$, we find $P=779$ for the MDL and $P=2694$ for the FPE, AIC and CAT.

Just as a trial we make a whitening with $P=3000$ on a single set of these data. The result is plotted in figure 10. The whitening is very good: in fact the parameter of flatness is 0.77 for the Durbin whitening algorithm and 0.83 for the LSL whitening algorithm.

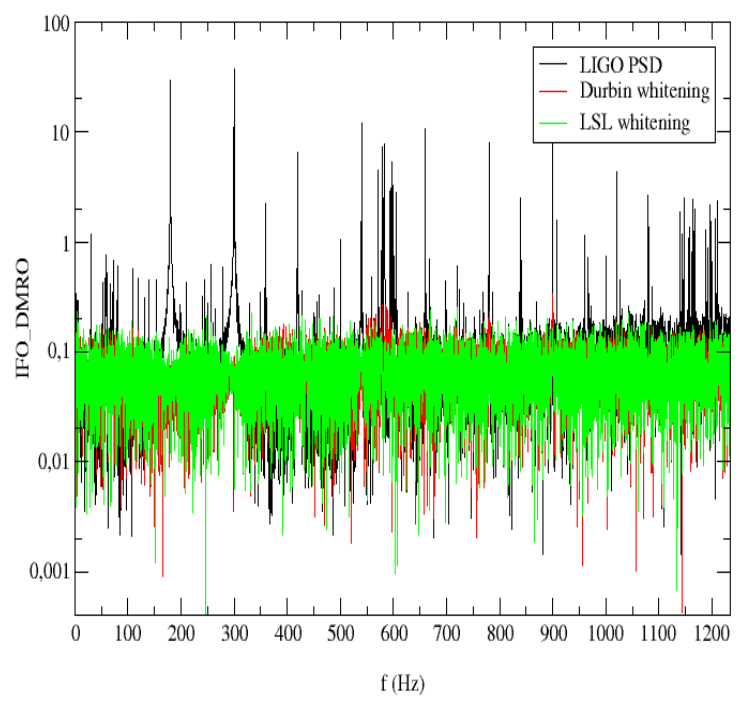

FIG. 10. PSD at the output of whitening filters with $P=3000$ for 18 nov94.2. frame data in the band $0-1233.5 \mathrm{~Hz}$.

This is a proof that we can succeed in whitening the data if we choose a sufficiently high number of parameters to compensate the fact that we have all-poles models and that we need a high number of poles to whiten broad peaks.

\section{B. 19nov94.2.frame}

We perform the same kind of analysis even on the data set 19nov94.2.frame.

We find the order of the filter using the results of the order selection criteria on a set of data long $\sim 6 \mathrm{~s}$, choosing $P=663$ that is the best order find by the MDL criterion (see table [V]).

\begin{tabular}{|c|r|r|r|}
\hline \hline MDL & AIC & FPE & CAT \\
\hline \hline 663 & 2351 & 2351 & 2351 \\
\hline \hline
\end{tabular}

TABLE IV. Order selection criteria for 19nov94.2.frame on the Nyquist interval. 
In figure 11 we showed the results of whitening filters on 19nov94.2.frame data at an order $P=663$.

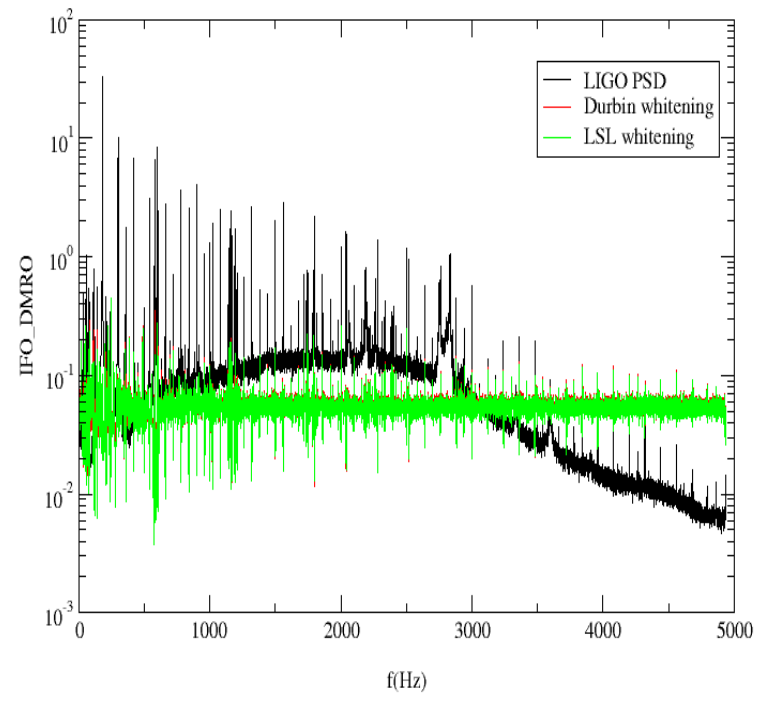

FIG. 11. 19nov94.2.frame: psd of input and output for whitening filters with $P=663$.

The kind of problem we met in the whitening results is the same as in 18nov94.2.frame, that is besides the several lines at high frequencies, it is difficult to whiten the low frequency broad peaks. A typical value of the flatness at the output of Durbin filter is 0.53 , whilst for the LSL filter is 0.55 .

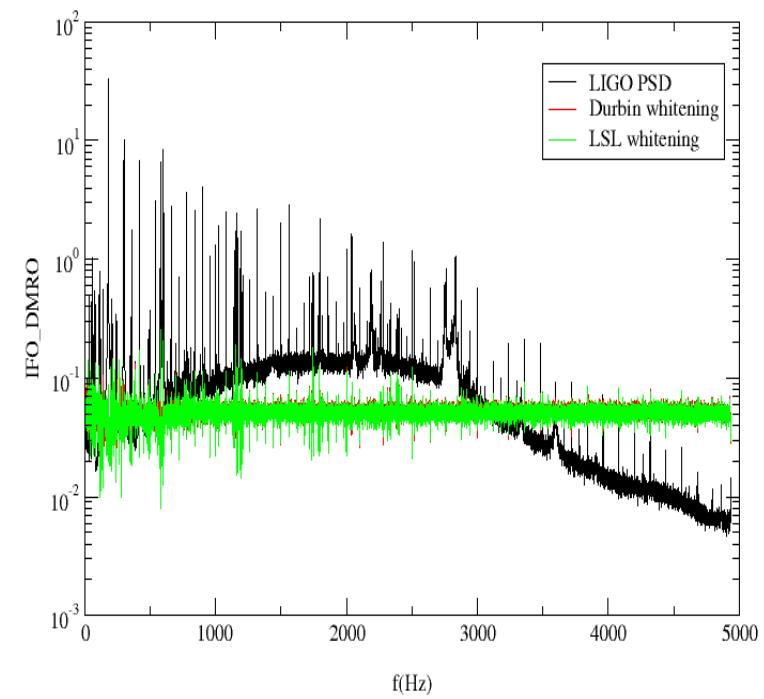

FIG. 12. 19nov94.2.frame: PSD of input and output for whitening filters with $P=2351$.
In figure 12 we report the whitening results obtained with filter of $P=2351$ order. The whitening is remarkably better .The values of the flatness are $\sim 0.6$ for the Durbin filter and $\sim 0.63$ for the LSL one.

\section{Whitening on a restricted band of frequencies}

We perform the whitening procedure also on this frame using a restricted band of frequencies. The selection order criteria give in this case $P=412$ for the MDL and $P=912$ for the others (see table $\mathrm{V}$ ).

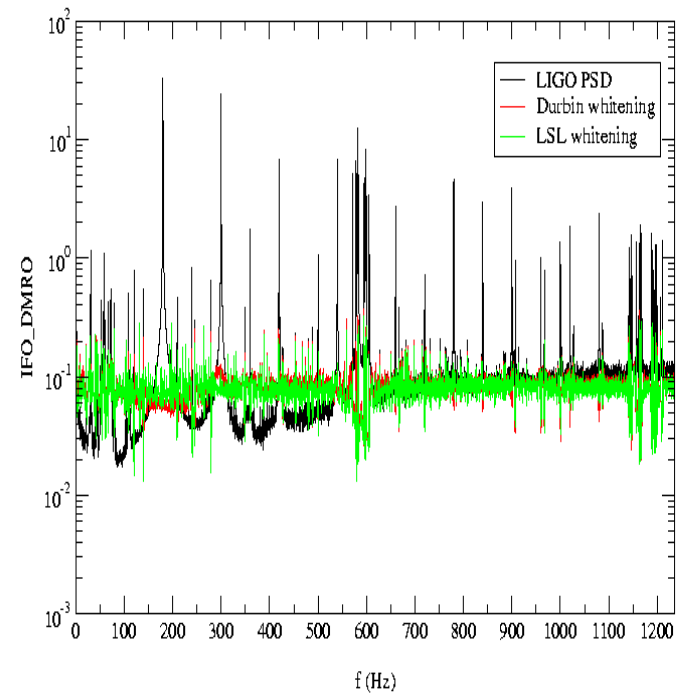

FIG. 13. 19nov94.2.frame: PSD of input and output for whitening filters on the band $0-1233.5 \mathrm{~Hz}$ with $P=412$.

\begin{tabular}{|r|r|r|r}
\hline \hline MDL & AIC & FPE & CAT \\
\hline \hline 412 & 912 & 912 & 912 \\
\hline \hline
\end{tabular}

TABLE V. Order selection criteria for 19nov94.2.frame on the band $0-1233.5 \mathrm{~Hz}$. 


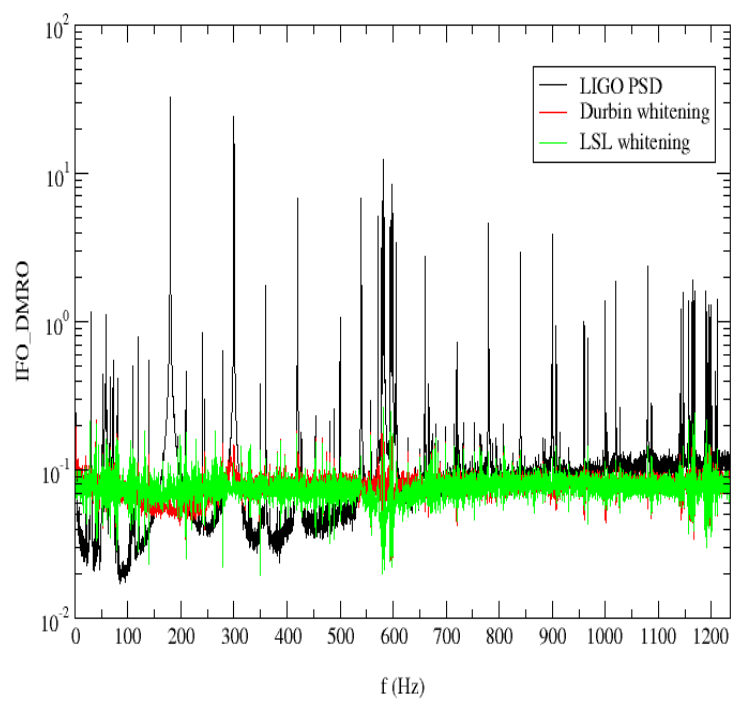

FIG. 14. 19nov94.2.frame: PSD of input and output for whitening filters on the band $0-1233.5 \mathrm{~Hz}$ with $P=912$.

The whitening filter with these order have been implemented on 30 samples of length $6 \mathrm{~s}$. The results are plotted in figures 1314.

The whitening with $P=912$ is good; in this case the typical value for the flatness parameter is 0.65 for Durbin filter and 0.7 for LSL one. We make the whitening, just as a trial, on this set of data using filter of $P=4096$. In figure 15 we plotted the results. The whitening procedure is good and the values of flatness is 0.66 for Durbin filter and 0.77 for LSL one. These values are not very different from the ones obtained with filters of order $P=912$.

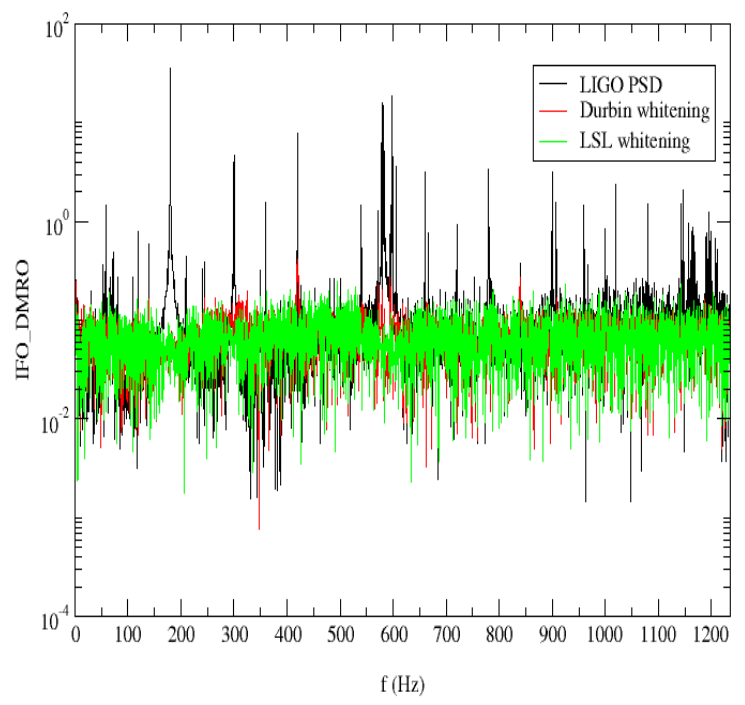

FIG. 15. 19nov94.2.frame: psd of input and output for whitening filters on the band $0-1233.5 \mathrm{~Hz}$ with $P=4096$.

\section{CONCLUSION}

We showed that we can obtain a whitened PSD on realistic interferometric data. In the two frames 18nov94.2 and 19nov94.2 of LIGO 40-meter interferometer we processed we found that, restricting our analysis to a band of frequencies and using a sufficiently high number of parameters, we can obtain whitened data in time domain on which we can apply our detection algorithms. It could be useful to statistically characterize the LIGO data to check out the presence of non stationary or non linearities in order to study the behavior of the whitening filters. Indeed we verified that the adaptive algorithm has a better performance with respect to the static algorithm so it could be useful if we have to face with non stationary data.

In whitening the LIGO 40-meter data we applied the filters directly to the data but, in principle, it could be useful before applying the whitening filters to cancel out the harmonics of $60 \mathrm{~Hz}$ [20 [37 [38. We want to underline that we apply these techniques to check their capability in whitening the power spectral density, but we could apply them in a more efficient way if we know in advance the kind of analysis we want to perform after the whitening, because we can choose to have a smoother level of whiteness or to analyze a narrow band of frequencies. The filters we use belong to the family of Kalman filter, but the dynamical law we suppose to underline the process is a general one, so in principle we can whiten all the spectral lines of the PSD. This could be advantageous if we want to perform the whitening in one step, because we know that the 'risk' of finding a periodic gravitational signal with an amplitude comparable to the ones of the violin modes or other spectral lines in the PSD is very low.

\section{ACKNOWLEDGMENTS}

The authors wish to thank the LIGO Laboratory for having made its prototype interferometer data from November 1994 available to the Virgo collaboration. They especially wish to acknowledge the LIGO authors in reference [1] [22] 23] who had a significant role in the acquisition of the data. Moreover the authors wish to thanks Albert Lazzarini for his courtesy and Andrea Vicere' for having made at their disposal these data and for very useful discussions. 
[1] Ando M et al 2000 Proc. 3rd E. Amaldi Conference (New York: AIP Proceedings) p 128

[2] Luck H et al 2000 Proc. 3rd E. Amaldi Conference (New York: AIP Proceedings) p 119

[3] Coles M W 2000 Proc. 3rd E. Amaldi Conference (New York: AIP Proceedings) p 101

[4] Abramovici et al 1992 Science 256 p 325

[5] Marion F 2000 Proc. 3rd E. Amaldi Conference (New York: AIP Proceedings) p 110

[6] Saulson P R 1994 Fundamentals of Interferometric Gravitational Wave Detectors World Scientific Pub Co ISBN:9810218206

[7] Blair D G (ed) 1993 The Detection of Gravitational Waves (Cambridge: Cambridge University Press)

[8] Barone M, Calamai G, Mazzoni M, Stanga R, Vetrano F (Eds) 2000 Proceedings of the International Summer School on Experimental Physics of Gravitational Waves (Singapore: World Scientific Pub)

[9] C. Bradaschia et al. 1999 Nucl. Instr. Meth. A 289 p 518

[10] G. Cagnoli et al., Phys. Lett. A, 255,pp 230-235, 1999

[11] B. J. Meers, Phys. Rev. D, 38 (8) 2317-2326, 1988

[12] Thorne K. S. and Winstein C. J. 1999 Phys.Rev.D 60 082001

[13] Hughes S. A and Thorne K. S. 1998 Phys.Rev.D 58 122002

[14] Cella G. Cuoco E 1997 VIR-NOT-PIS-1390-099 (Internal Note)

[15] Beccaria M et al 1998 Classical and Quantum Gravity 15 p 3339

[16] Cella G Off-line Subtraction of Newtonian Noise 1999 Proc. of 34 Rencontre de Moriond Conf. (In press)

[17] Cagnoli G. et al 1998 Phys. Lett. A 237 p 21

[18] V. B. Braginsky, Y. Levin, S. Vyatchanin, Measur. Sci.Tech., 10 ,1999, pp 598-606

[19] L.S. Finn and S. Mukherjee, gr-qc/0005061

[20] A. M. Sintes, B. F. Schutz, "Proceedings of the 2nd workshop on Gravitational Wave Data Analysis". Edts. M. Davier and P. Hello; Editions Frontières, pp. 255-260 (1998)

[21] , E. Chassande-Mottin, S. V. Dhurandhar,gr-qc/0004075

[22] Abramovici et al 1996 Phys. Lett. A 218 p 157

[23] B. Allen et al 1999 Phys. Rev. Lett. 83 p 1498

[24] Kay, Modern spectral estimation:Theory and Application, Prentice Hall, Englewood Cliffs, 1988

[25] Monson H. Hayes, Statistical digital signal processing and modeling, Wiley, 1996

[26] Charles W. Therrien, Discrete Random Signals and Statistical Signal Processing, Prentice Hall, 1992

[27] E. Cuoco, G. Calamai, L. Fabbroni, G. Losurdo, M. Mazzoni, R. Stanga, F. Vetrano Classical and Quantum Gravity 18 (2001) 1727

[28] L. A. Zubakov V. D. Wainstein. Extraction of signals from noise. Prentice-Hall, Englewood Cliffs, 1962.

[29] T.Pradier et al., Int.J.Mod.Phys. D9 (2000) 309-314

[30] Bruce Allen, GRASP: a data analysis package for gravitational wave detection, 1999

[31] B. Allen, P. Brady, Quantization noise in Ligo interferometer, Ligo internal note, 1997

[32] S. Thomas Alexander, Adaptive Signal Processing, Springer-Verlag, 1986
[33] E. Parzen, An approach to Time series modeling: determining the order of approximating autoregressive schemes, in "Multivariate Analysis", North Holland, 1977

[34] Simon Haykin, Adaptive Filter Theory, Prentice Hall, 1996

[35] M.Beccaria, E.Cuoco, G.Curci, Adaptive System Identification of VIRGO-like noise spectrum. Note II., VIR-NOT-PIS-1390-096, 1997; M.Beccaria, E.Cuoco, G.Curci, Adaptive System Identification of VIRGO-like noise spectrum, Proceeding of Second Amaldi Conference, 1997, World Scientific

[36] E. Cuoco, Whitening of noise power spectrum. Test on LIGO 40-meter interferometer data, VIR-NOT-FIR1390-145

[37] B. Widrow and S. D. Stearns, Adaptive Signal Processing, Prentice Hall, 1985.

[38] R. Flaminio, Monitoring and subtraction of $50 \mathrm{~Hz}$ lines in read-out noise, GWDAW 1999, Roma 